\author{
Supporting Information
}

\title{
Atomic Layer Densification of AIN \\ Passivation Layer on Epitaxial Ge for \\ Enhancement of Reliability and Electrical \\ Performance of high-K Gate Stacks
}

Chin-I Wanga, Teng-Jan Chang ${ }^{\mathrm{a}}$, Yu-Tung Yin ${ }^{\mathrm{a}}$, Yu-Sen Jiang ${ }^{\mathrm{a}}$, Jing-Jong Shyue ${ }^{\mathrm{b}}$ and Miin-Jang Chen ${ }^{\mathrm{a},}$

${ }^{a}$ Department of Materials Science and Engineering, National Taiwan University, Taipei, Taiwan, R.O.C.

${ }^{\mathrm{b}}$ Research Center for Applied Science, Academia Sinica, Taipei, Taiwan, R.O.C.

*Email: mjchen@ntu.edu.tw 


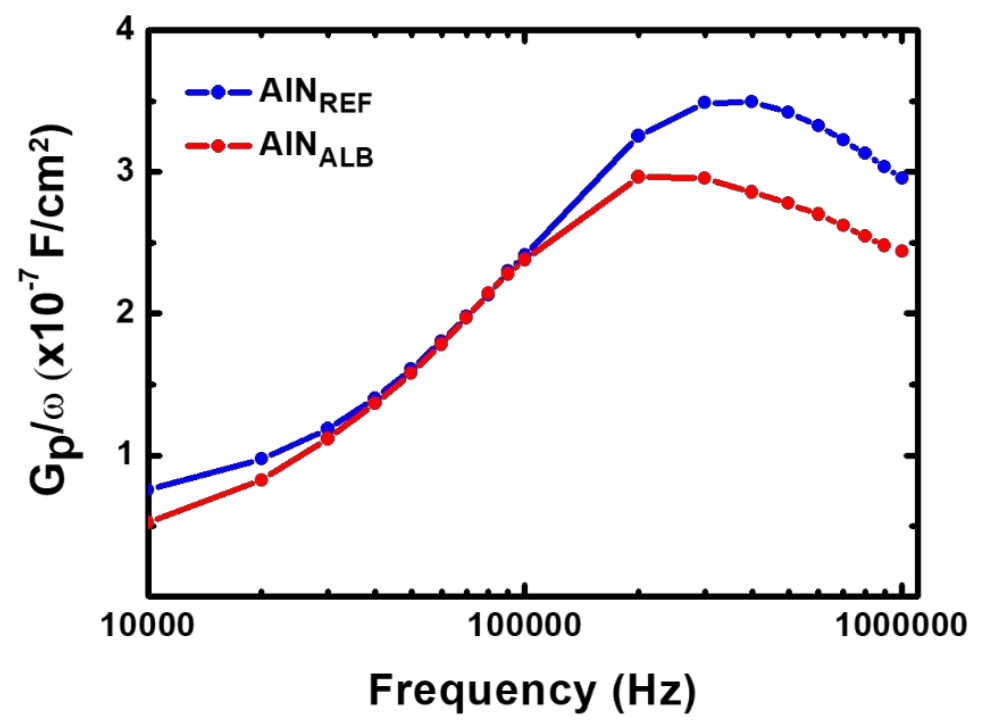

Figure S1. The equivalent parallel conductance over the angular frequency $\left(G_{p} / \omega\right)$ versus the frequency of the $\mathrm{AlN}_{\mathrm{REF}}$ and $\mathrm{AlN} \mathrm{N}_{\mathrm{ALB}} \mathrm{MOS}$ capacitors. The $D_{i t}$ can be extracted according to the formula $q D_{i t}=2.5\left(G_{p} / \omega\right)_{\max }$ ( $q$ is the elementary charge). It can be seen that the $\operatorname{AlN}_{\mathrm{ALB}}$ MOS capacitor has smaller $\left(G_{p} / \omega\right)_{\max }$, corresponding to a smaller $D_{i t}$ value. 

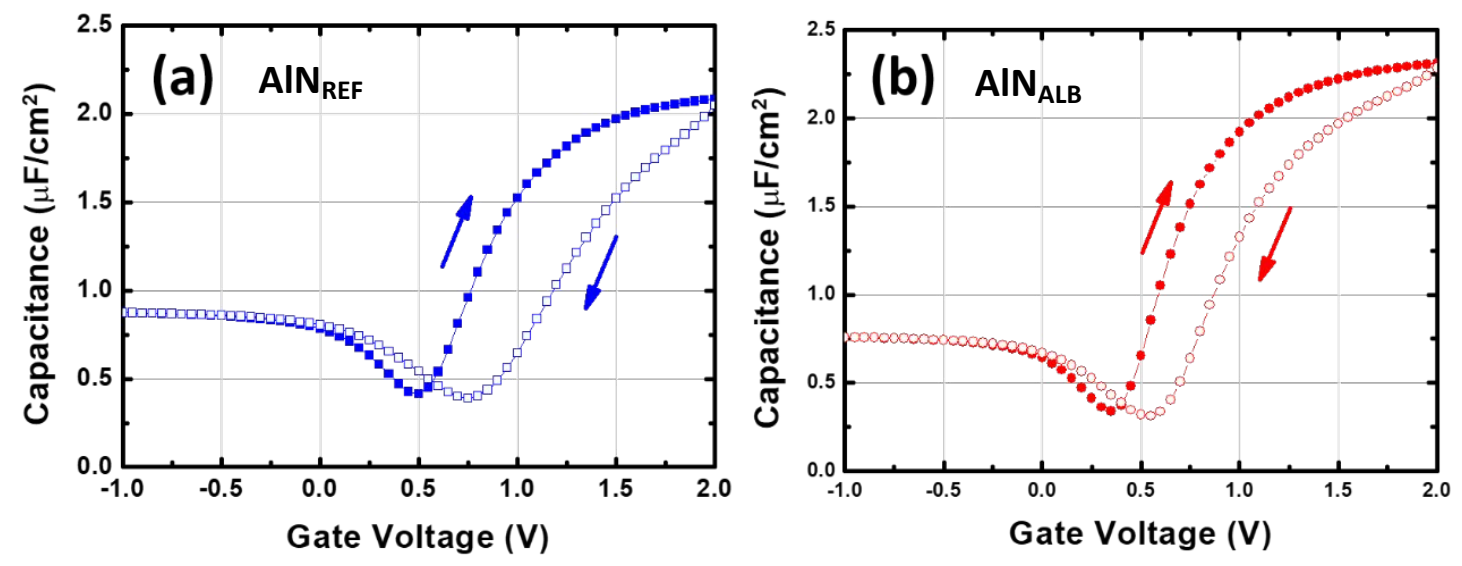

Figure S2. The $C$ - $V$ hysteresis curves of the (a) $\mathrm{AlN}_{\mathrm{REF}}$ and (b) $\mathrm{AlN}_{\mathrm{ALB}} \mathrm{MOS}$ capacitors at the frequency of $1 \mathrm{MHz}$. The hysteresis window is determined by the $V_{F B}$ difference between the forward and backward scans. A smaller hysteresis window was observed in the $\mathrm{AlN}_{\mathrm{ALB}} \mathrm{MOS}$ capacitor, which can be attributed to a lower slow trap density. 


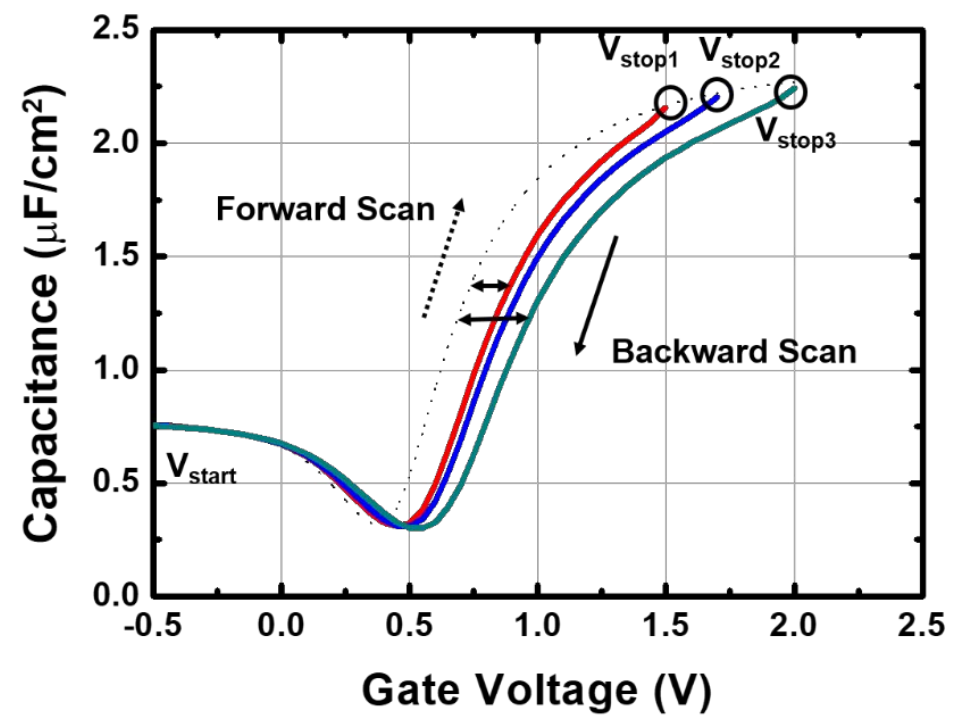

Figure S3. Extraction of $\Delta N_{s t}$ from the $C-V$ measurements. The minimum gate

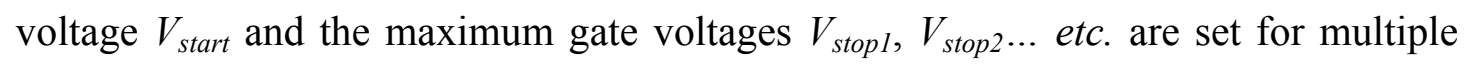
forward and backward $C-V$ sweeps. $\Delta N_{s t}$ can be evaluated using the expression $q \Delta$ $N_{s t}=C_{o x} \Delta V_{h y s}$, where $q$ is the elementary charge, $C_{o x}$ is the accumulation capacitance, and $\Delta V_{h y s}$ is the $V_{F B}$ difference between the forward and backward scans, respectively. 

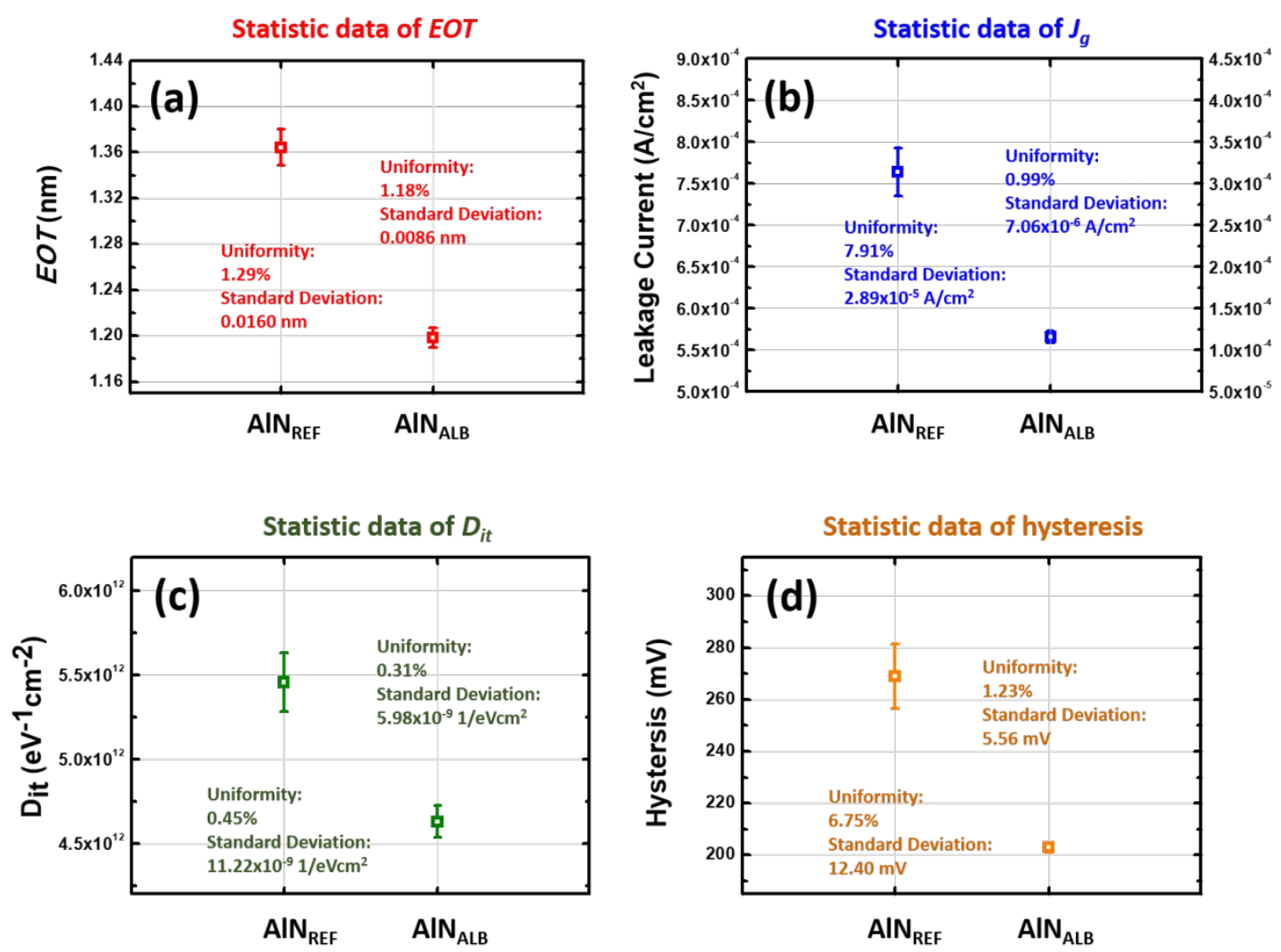

Figure S4. The statistical data of the (a) EOT, (b) $J_{g}$, (c) $D_{i t}$, and (d) hysteresis of the $A 1 N_{\text {REF }}$ and $\mathrm{AlN}_{\mathrm{ALB}}$ MOS capacitors, in which the error bar indicates one standard deviation. All the statistical data of the EOT, $J_{g}, D_{i t}$, and hysteresis reveal no overlap between the $A l N_{\mathrm{REF}}$ and $\mathrm{AlN} \mathrm{N}_{\mathrm{ALB}}$ samples. In addition, the uniformities and the standard deviations of the EOT, $J_{g}, D_{i t}$, and hysteresis for the $\mathrm{AlN}_{\mathrm{ALB}}$ sample are superior to those for the $A 1 N_{\text {REF }}$ MOS capacitor. Hence the outcomes manifest the improved electrical performance of the AIN passivation layer by the ALB treatment. 